\title{
Electrical Impedance Tomography During Mechanical Ventilation
}

\author{
Brian K Walsh PhD RRT-NPS FAARC and Craig D Smallwood RRT
}

\author{
Introduction \\ Application of Lung Electrical Impedance Tomography \\ PEEP Titration \\ Effects of Position Changes \\ Effects of Mode of Ventilation \\ Assessment of Regional Distribution of Ventilation \\ Quantification of Pulmonary Edema \\ Available Technologies \\ Limitations \\ Conclusions
}

\begin{abstract}
Electrical impedance tomography (EIT) is a noninvasive, non-radiologic imaging modality that may be useful for the quantification of lung disorders and titration of mechanical ventilation. The principle of operation is based on changes in electrical conductivity that occur as a function of changes in lung volume during ventilation. EIT offers potentially important benefits over standard imaging modalities because the system is portable and non-radiologic and can be applied to patients for long periods of time. Rather than providing a technical dissection of the methods utilized to gather, compile, reconstruct, and display EIT images, the present article seeks to provide an overview of the clinical application of this technology as it relates to monitoring mechanical ventilation and providing decision support at the bedside. EIT has been shown to be useful in the detection of pneumothoraces, quantification of pulmonary edema and comparison of distribution of ventilation between different modes of ventilation and may offer superior individual titration of PEEP and other ventilator parameters compared with existing approaches. Although application of EIT is still primarily done within a research context, it may prove to be a useful bedside tool in the future. However, head-to-head comparisons with existing methods of mechanical ventilation titration in humans need to be conducted before its application in general ICUs can be recommended. Key words: electrical impedance tomography; regional distribution of ventilation; lung imaging; mechanical ventilation. [Respir Care 2016;61(10):1417-1424. (C) 2016 Daedalus Enterprises]
\end{abstract}

\section{Introduction}

Electrical impedance tomography (EIT) uses electrical currents to assess the conductivity distribution within the

\footnotetext{
Dr Walsh and Mr Smallwood are affiliated with the Division of Critical Care Medicine, Department of Anesthesiology, Perioperative and Pain Medicine, Boston Children's Hospital, Boston, Massachusetts and Harvard Medical School, Boston, Massachusetts. Mr Smallwood is also affiliated with the Department of Bioengineering, Northeastern University, Boston, Massachusetts.
}

body from voltage measurements of its surface. This concept was first described as a method to explore subterranean mineral deposits in the early $1900 \mathrm{~s}^{1}$ and later adapted

\footnotetext{
The authors have disclosed a research relationship with Dräger Medical in which Dräger supplied research equipment but no financial support.

Dr Walsh presented a version of this paper at the 31st New Horizons Symposium: Monitoring at the AARC Congress 2015, held November 7-10, 2015, in Tampa, Florida.
} 
for medical use. When we breathe, volumes of electrically insulating air move in and out of the lungs. This volume changes within the lungs, which produces conductivity changes that can be detected by EIT. Barber and Brown introduced EIT to the medical community in the early 1980 s. $^{2}$ From there, a wide spectrum of applications in medicine ranging from gastric emptying, brain function, and breast imaging to lung function have been explored. Lung imaging is an ideal application of EIT because human ventilation exhibits large changes in volume (and therefore electrical conductivity) and also because of the fact that the lungs are relatively close to the surface (where measurements are obtained). Imaging of ventilation could become a key clinical application of EIT.

Early EIT devices were fairly limited due to poor sensitivity and signal interference in the clinical setting. ${ }^{2}$ After years of waxing and waning interest from a few investigators and companies, a renewed interest in ventilation technology helped to resolve many of these shortcomings. As with any new modality, EIT and its clinical utility and application need to be methodically explored. Rather than providing a technical dissection of the methods utilized to gather, compile, reconstruct, and display EIT images, the present article seeks to provide an overview of the clinical application of this technology as it relates to monitoring mechanical ventilation and providing decision support at the bedside.

Patients who require positive-pressure ventilation to reverse inadequate gas exchange are at risk of ventilatorinduced lung injury, primarily through overdistention (volutrauma) or repeated opening and closing of gas exchange units with each breath (tidal recruitment) leading to atelectrauma. This can lead to an inflammatory cascade of events known as biotrauma. ${ }^{3,4}$ This awareness has triggered additional lung-protective research, including the use of EIT to guide mechanical ventilation. Key components of lung protection are reducing not only global overdistention or collapse, but regional collapse as well, while maintaining adequate gas exchange. Before the use of EIT, radiographic images were the only way to look at regional distribution of ventilation.

EIT capitalizes on changes in electrical impendence between air-filled versus tissue or fluid-filled spaces to characterize and quantify regional distribution of lung volume at the bedside. This technology has been validated in animal $^{5}$ and human 6,7 studies. EIT utilizes a series of elec-

Correspondence: Brian K Walsh PhD RRT-NPS FAARC, Critical Care Medicine, Department of Anesthesiology, Perioperative and Pain Medicine, MSICU Office, Bader 634, Children's Hospital, 300 Longwood Avenue, Boston, MA 02115.E-mail: Brian.walsh@childrens.harvard.edu.

DOI: $10.4187 /$ respcare.04914

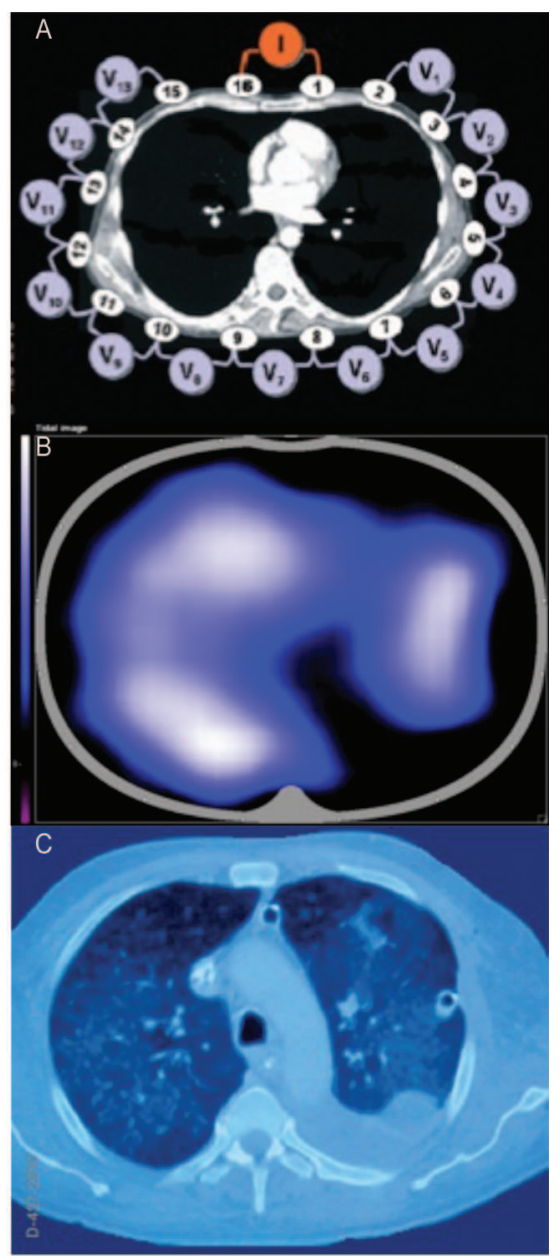

Fig. 1. Electrical impedance tomographic (EIT) images are created using a series of electrodes placed across the chest, each of which send and receive electrical impulses with one another (A). Shown are a functional EIT image (white indicates highest volume changes, non-ventilated regions are dark blue) (B) and computed tomography scan (C) from a patient with a pleural effusion and significant lung disease. Courtesy Dräger.

trodes (typically 16 or 32) arranged circumferentially around the patient's thorax (Fig. 1A). Small currents, which are undetectable to the patient, are passed between the electrodes, and impedance is measured between and among the electrode array. Through a complex interrogation and manipulation of these impedance values, a 2-dimensional image is formed (Fig. 1B) and has been shown to correlate with clinical and radiographic changes in subjects (Fig. 1C). ${ }^{6}$ The ability to estimate lung volume and regional distribution of ventilation noninvasively and in real-time may give us valuable insight into never before understood effects of mechanical ventilation changes.

\section{Application of Lung EIT}

Current strategies to provide lung-protective ventilation rely on avoiding conditions associated with lung injury. 
There is growing interest in developing individualized mechanical ventilation treatment plans. The use of EIT has demonstrated reasonable agreement with traditional imaging modalities for assessing regional lung volume changes in animals and humans. ${ }^{5,8,9}$ Although radiological and EIT images both provide information about regional distribution of air in the lungs, the image characteristics provided by these modalities are quite different. Radiological images of the lung provide useful information on the distribution of ventilation and proportion and location of alveolar collapse but are limited because only a discrete sample in time is obtained; specifically, changes that occur dynamically or at any other time point would be missed by traditional imaging modalities. EIT images typically reflect changes in lung inflation, which means that EIT displays ventilated lung regions rather than morphological or anatomical structures of the lung. In the literature, this is referred to as functional EIT. The ideal clinical monitor for mechanically ventilated patients would provide measures of ventilation and perfusion as well as some metric of the spatial agreement between the two. Indeed, EIT has been used as a tool to assess changes not only in ventilation but in pulmonary perfusion as well. ${ }^{10-12}$ However, a robust clinical trial assessing the accuracy of perfusion mapping by EIT and its clinical utility needs to be conducted. Nonetheless, the regional information contained in EIT and computed tomography (CT) images are closely related to each other when pathological conditions, such as pleural effusion or atelectasis, lead to non-aerated and non-ventilated (non-functional) lung regions. Whereas CT images display lungs regions with trapped air (eg, pneumothorax) in black because of the large air content, most EIT systems also display those regions in black because they are not ventilated. ${ }^{13,14}$ Conversely, a CT image may indicate a region of consolidated lung tissue in a white color because of the high fluid content, whereas this region might be displayed in the EIT image in black or dark blue if this region is not ventilated or is only partially ventilated. ${ }^{15}$ See Figure 2 for details.

\section{PEEP Titration}

The application of PEEP is known to prevent alveoli collapse and homogenize ventilation. Setting the optimal and individualized PEEP levels in patients with and without lung injury to avoid atelectasis and alveolar strain is difficult at best. Several methods have been proposed to titrate PEEP: low-flow pressure-volume curves, ${ }^{16} \mathrm{CT},{ }^{17}$ esophageal monometry, best pulmonary compliance, ${ }^{18}$ and standardized tables based on gas exchange or oxygen requirements (PEEP-F $\mathrm{IO}_{2}$ table). ${ }^{19,20}$ With the exception of $\mathrm{CT}$, none of the aforementioned have the ability to detect regional inhomogeneous distribution of ventilation. Although PEEP adjustments based on PEEP- $\mathrm{F}_{\mathrm{IO}_{2}}$ charts have

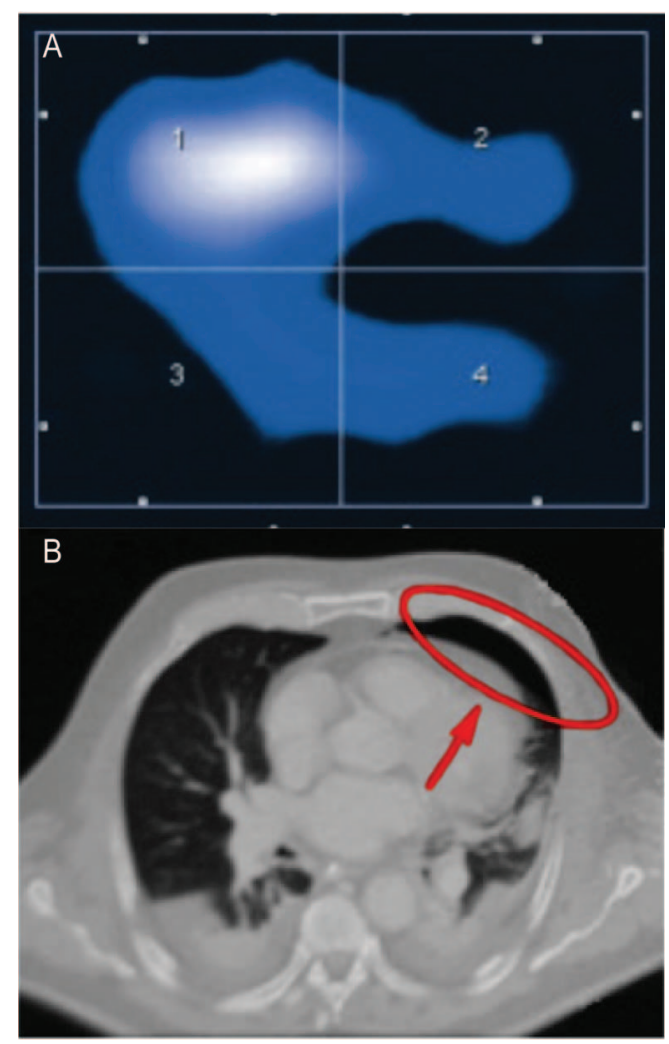

Fig. 2. A: Functional electrical impedance tomographic tidal image of a patient with a pneumothorax. Note the black in the upper right of box 2; this means that impedance is not changing in that region, and therefore no volume is changing. $\mathrm{B}$ : Corresponding computed tomogram demonstrating a pneumothorax (circled area) in the same region. Courtesy Dräger.

been shown to offer a weak correlation with lung recruitability $\left(\mathrm{R}^{2}=0.29\right)$ in adult subjects with ARDS, the majority of recruitability of a patient cannot be explained with standard methods of PEEP titration. Algorithms for the quantification of recruitable alveolar collapse and overdistention have been demonstrated with EIT and have shown agreement with CT. However, these methods must be compared with standard methods, including PEEP- $\mathrm{F}_{\mathrm{IO}_{2}}$ charts, pressure-volume curves, and esophageal monometry, before a clear benefit of EIT-derived lung mechanics can be asserted.

EIT offers a radiation-free technique of quantifying regional distribution of ventilation at the bedside. Pulletz et $\mathrm{a}^{21}$ describe the use of regional opening pressure and regional closing pressure based on the simultaneous measurement of airway pressure and EIT impendence change that can reduce inhomogeneous distribution of ventilation in healthy and lung-injured subjects. Our group was able to identify lung opening, overdistention, and collapse within a stair-step lung recruitment protocol. ${ }^{22}$ Figure 3 demonstrates a baseline setting of PEEP of $15 \mathrm{~cm} \mathrm{H}_{2} \mathrm{O}$ that climbs to $30 \mathrm{~cm} \mathrm{H}_{2} \mathrm{O}$ in a patient with ARDS. Pixel coloring is 

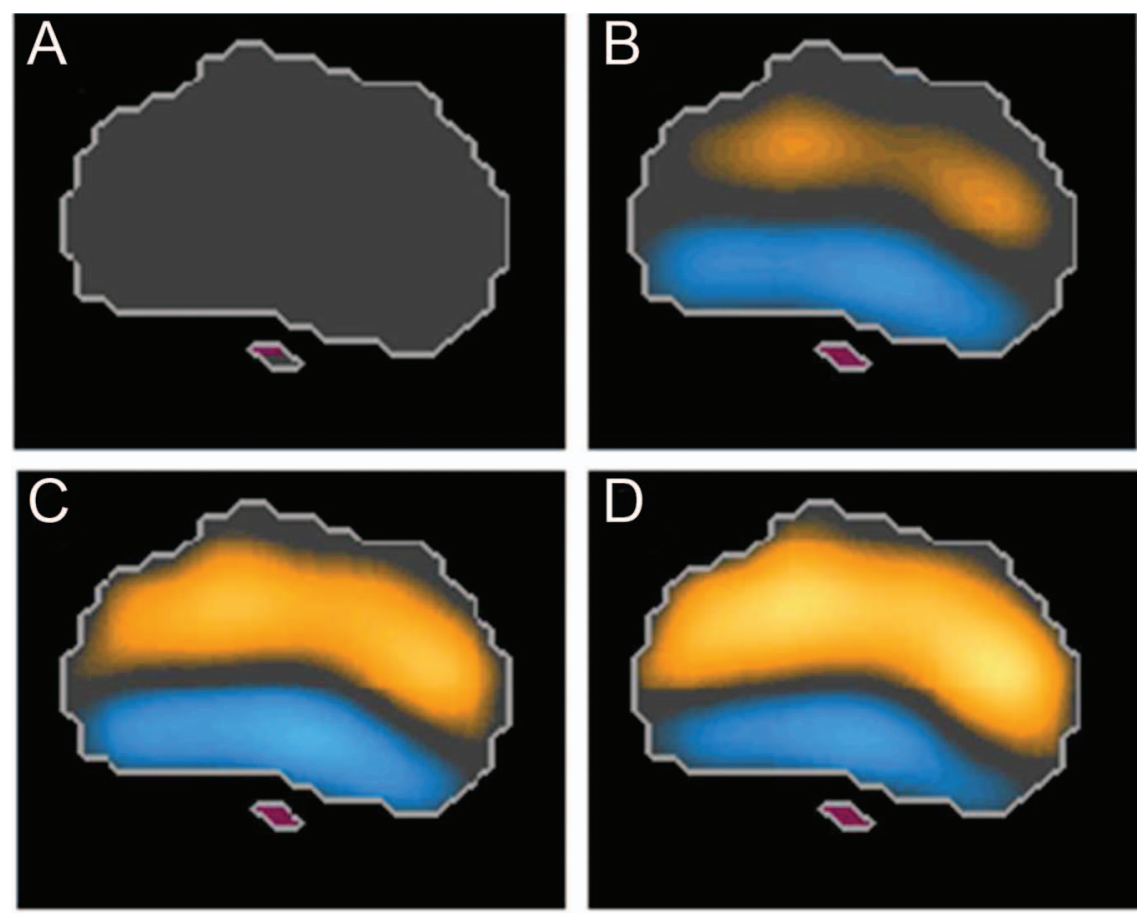

Fig. 3. Baseline electrical impedance tomographic tidal images in which changes in impedance are compared, using the Costa et al23 definition of alveolar overdistension (orange) and opening (blue).

often used to describe changes visually. Most EIT images have a color legend to help guide interpretation. Figure 3 utilizes the Costa et $\mathrm{al}^{23}$ definition of alveolar overdistention (orange) and opening (blue). As you will see, overdistention (orange) starts with the second step (Fig. 3B) but may be outweighed by the benefit of volume recruited. At step 3, a PEEP of $25 \mathrm{~cm} \mathrm{H}_{2} \mathrm{O}$ (Fig. 3C), you will see that overdistention and recruitment appear to be balanced, with slightly more overdistention. However, in step 4, a PEEP of $30 \mathrm{~cm} \mathrm{H}_{2} \mathrm{O}$, it is clear that overdistention is occurring in the majority of this window of the lung. Overdistention is often required of the compliant sections of the lung to recruit the noncompliant diseased sections. Although lung recruitments maneuvers have been shown to be beneficial in improving gas exchange, the stress and strain required are a concern. EIT can help quantify gains (recruitment) and losses (overdistention or de-recruitment) at the bedside and in real time when gas exchange improvements can be misleading; thus, the clinician would be able to prioritize recruitment versus overdistention for select patients.

\section{Effects of Position Changes}

Properly positioning patients who require mechanical ventilation is one of several strategies demonstrated to reduce complications of mechanical ventilation. ${ }^{24}$ Headof-bed elevation has been part of a ventilator-associated pneumonia prevention bundle for years. ${ }^{25}$ EIT has been used to evaluate whether global and regional end-expiratory lung volumes improve by the degree of elevation. Spooner et $\mathrm{al}^{26}$ found that head-of-bed elevation increases end-expiratory lung volumes and recommended, unless contraindicated, all mechanically ventilated patients should have their head of bed elevated. Figure 4 demonstrates the improvements in impedance by regions and the degree of elevation. Compared to standard head of bed elevation, Bein et a ${ }^{27}$ showed that tilting ARDS subjects 60 degrees on their left or right sides did not effect distribution of ventilation. In such cases, the role of EIT serves as a metric to assess the functional effect on the pulmonary system in response to position changes.

\section{Effects of Mode of Ventilation}

Newer modes of ventilation have been cleared by the FDA, often through substantial similarity to a predicate device. However, there are extremely limited data that would substantiate a claim that one mode of ventilation is superior to another. Often, regional distribution of ventilation is not evaluated as part of the FDA clearance process. Mauri et $\mathrm{al}^{28}$ compared the involvement of dependent (dorsal) lung regions of ARDS subjects ventilated with pressure support ventilation. They concluded that an increase in PEEP by $5 \mathrm{~cm} \mathrm{H}_{2} \mathrm{O}$ improved regional distribution of ventilation, improving compliance and leading to a 


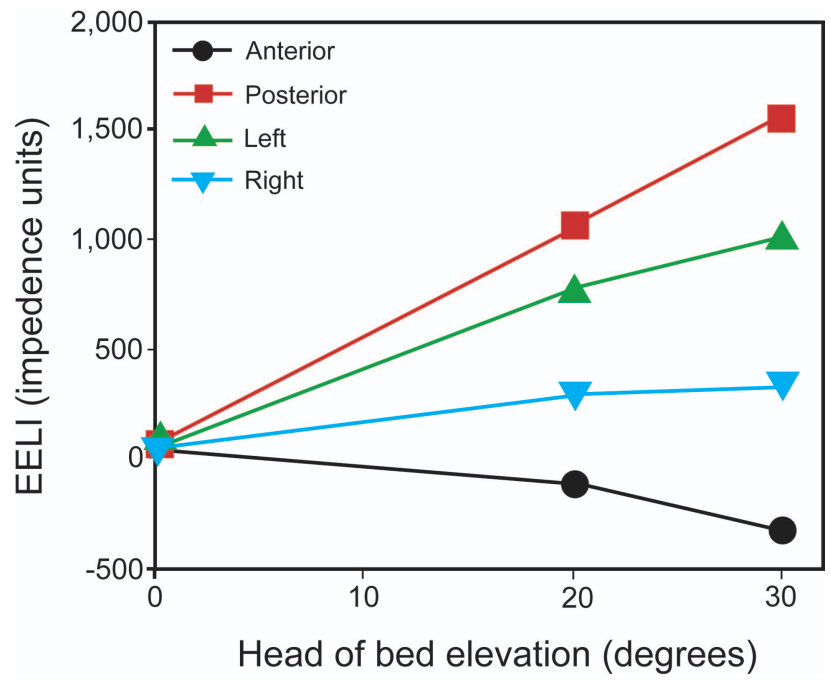

Fig. 4. Changes from baseline in mean end-expiratory lung impedance (EELI) by lung region and at each head-of-bed level. From Reference 26.

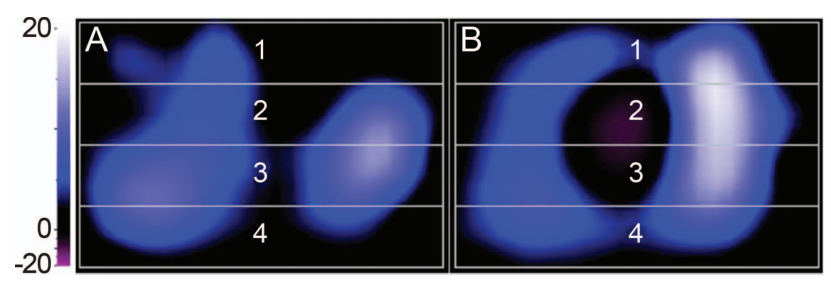

Fig. 5. Differences in regional distribution of ventilation within the same patient, using pressure support ventilation $(A)$ and neurally adjusted ventilatory assist (B). In this patient, there appears to be a more homogeneous distribution of ventilation using neurally adjusted ventilatory assist ventilation.

decrease in pressure support ventilation by $4 \mathrm{~cm} \mathrm{H}_{2} \mathrm{O} .^{28}$ Likewise, observations by Blankman et al ${ }^{29}$ showing that lower support, both in pressure control and neurally adjusted ventilatory assist mode, provided improved ventilation in dependent regions using regional distribution of ventilation as assessed by EIT as the outcome.

We are currently evaluating the distribution of ventilation between neurally adjusted ventilatory assist and pressure support ventilation in a pediatric crossover trial in which EIT imaging is assisting in our evaluation of the effects of modes of ventilation (ClinicalTrials.gov registration NCT-01504373). Figure 5 demonstrates the differences in regional distribution of ventilation within the same patient. EIT may allow us to individualize care and utilize modes of ventilation that produce the most objective improvement.

\section{Assessment of Regional Distribution of Ventilation}

Patients with lung injury suffer from inhomogeneous conditions, differing properties of dependent and non- dependent regions, and the effects of positioning and shifts in transpulmonary pressure. The varying and often unmeasurable conditions place the clinician at a disadvantage to completely understand which condition is occurring. When patients spontaneously breathe while receiving positivepressure ventilation, negative pleural pressure increases transpulmonary pressure. Because spontaneous ventilation often augments gas exchange, minimizes muscle atrophy, and improves respiratory system compliance, it is traditionally encouraged. However, in the injured lung, this pleural pressure is not uniformly transmitted and can result in a pendelluft phenomenon from non-dependent to dependent lung regions without changes in tidal volume, meaning that without EIT or CT scan, most clinicians would be blind to this phenomenon. During lung-protective ventilation with strict adherence to limiting tidal volumes, a strong inspiratory effort may be injurious by causing local overdistention in the dependent lung units. ${ }^{30}$ The pendelluft effect may be the reason why, in mild lung injury, spontaneous breathing is beneficial, yet in severe lung disease, spontaneous breathing may be harmful. ${ }^{31}$ Further, this hypothesis has been tested in a well-performed randomized controlled trial published by Papazian et $\mathrm{al}^{32}$ demonstrating that early paralysis in severe ARDS is beneficial. EIT has the potential to identify in which patients pendelluft is occurring.

EIT has been shown in animal models and human case reports to be an accurate indicator of pneumothoraces. ${ }^{13,33,34}$ More recently, EIT-derived parameters have been used to differentiate atelectatic, overdistended, and adequately recruited lung in different lung regions by a number of investigators..$^{5,21,22,35-41}$ Despite a large body of literature, only recently have EIT-derived indices has been shown to improve outcomes in an experimental model of lung injury. Wolf et al $^{42}$ developed an EIT-guided mechanical ventilation strategy that demonstrated promise in an animal model. Through this experimental model, they were able to demonstrate that EIT-guided ventilation was superior to a national and standardized ventilation protocol called the Acute Respiratory Distress syndrome Network (ARDSNet) Ventilator Protocol.43-46 EIT-guided ventilation produced improved gas exchange and improved aeration as corroborated by CT imaging and had no adverse hemodynamic impact. Importantly, lung histopathology showed reduced lung injury in the EIT-guided group compared with the control group. In an experimental model of lung injury, EIT offers regional ventilation assessment to allow the clinician to optimize treatment with mechanical ventilation.

Another way to assess changes in regional distribution of ventilation is through the use of $\Delta$ impedance over time graphs. Figure 6 is a graphical display of a patient who is receiving tidal ventilation (baseline phase noted in figure). The patient receives a recruitment maneuver and returns to 


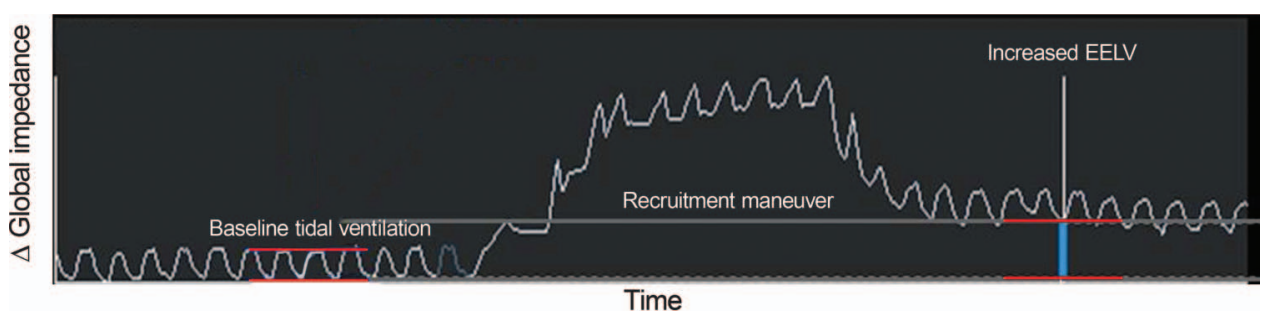

Fig. 6. Graphical display of global impedance changes over time. Following the baseline assessment, the patient is exposed to a recruitment maneuver followed by a reassessment of distribution of ventilation. The reassessment shows the increase in end-expired lung volume (EELV) following the recruitment maneuver. Courtesy Dräger.

Table 1. Known Manufacturers of Electrical Impedance Tomography Technology, System Names, Electrode Configurations, and How They Measure Tidal Volume

\begin{tabular}{lll}
\hline \hline Manufacturer & \multicolumn{1}{c}{ EIT System } & \multicolumn{1}{c}{ Pneumotachometer } \\
\hline Dräger Medical & PulmoVista 500 (16-electrode belt) & Dräger Evita or V500 data connection \\
Swisstom & BB $^{2}(32$-electrode belt) & Maquet (Europe) and SALVIA elisa 800 VIT \\
Timpel & Enlight 1800 (32-electrode bipartite belts) & Built-in pressure differential
\end{tabular}

EIT $=$ electrical impedance tomography; VIT $=$ ventilator integrated tomography

the previous settings. As you see, the recruitment maneuver resulted in an increase in end-expired lung volumes relative to the baseline tidal ventilation phase (blue line). This can be explored globally as in Figure 6 or by region to determine the effect of a change in mechanical ventilation, position, or procedure, such as a recruitment maneuver, chest tube, or compartment decompression. This is often used within research protocols because it is more objective than EIT images.

\section{Quantification of Pulmonary Edema}

Until recently, the assessment of pulmonary edema has required invasive measurements or is often unreliable. Chest radiographs are often used to estimate pulmonary fluid content but are only capable of detecting extravascular lung water $>38 \%$ of normal. ${ }^{47}$ In an experimental animal model, a novel noninvasive technique using changes in thoracic EIT with lateral body rotation was able to estimate extravascular lung water. ${ }^{48}$ This lung water EIT ratio holds promise in assisting clinicians with fluid resuscitation and management while minimizing ventilator-induced lung injury.

\section{Available Technologies}

To our knowledge, there are 3 manufacturers of EIT devices. None are cleared by the FDA for use in the United States. Table 1 describes the 3 manufacturers of EIT systems, the number of electrodes they use, and how they measure tidal volumes: the PulmoVista 500 (Dräger Med- ical, Lübeck, Germany), BB² (Swisstom AG, Landquart, Switzerland), and Enlight 1800 (Timpel, São Paulo, Brazil). The PulmoVista 500 uses 16 electrodes to measure the voltages utilized for image reconstruction, whereas the $\mathrm{BB}^{2}$ and Enlight 1800 employ a 32-electrode array. For the PulmoVista 500, mathematical simulations based on this electrode arrangement demonstrate a spatial resolution of $15 \%$ of the thoracic diameter; however, the resolution decreases to $20 \%$ toward the center of the body. Whereas CT scanners typically provide images consisting of $512 \times 512$ or more pixels, EIT images from the PulmoVista 500 only consist of $32 \times 32$ pixels, 256 times fewer pixels than CT images. ${ }^{2}$ Although the 16 electrode array is limited in the number of pixels that can be reconstructed for each image, its use is the most investigated and accepted in the field. The $\mathrm{BB}^{2}$ is unique, since the electrode belt offers onboard computation, permitting filtering and processing for image reconstruction in a small package. The Timpel Enlight system has recently been shown to be useful in the detection and quantification of edema in a model of lung injury. 48

\section{Limitations}

Although EIT appears to offer important advantages over traditional methods of assessing the adequacy of mechanical ventilation, widespread adoption of the technology has not occurred. Importantly, large clinical trials demonstrating the superiority of EIT over traditional methods of mechanical ventilation titration have not been completed. Until such time that substantial evidence exists 
demonstrating the superiority of EIT (in humans), it will probably remain a research tool for most institutions. Further, the availability of devices is extremely limited; no such device is cleared by the FDA, and therefore are not available for purchase in the United States. Another important limitation is the spatial resolution of currently available (for research purposes) devices. EIT offers only a small fraction of the resolution obtained during CT. Another important limitation is the reliance on accurate placement of the electrodes on the chest; there are limited data assessing the feasibility of long-term application of the belts and electrodes used during EIT.

\section{Conclusions}

EIT has been shown to be useful in the detection of pneumothoraces, quantification of pulmonary edema, and comparison of distribution of ventilation between different modes of ventilation and may offer superior individual titration of PEEP and other ventilator parameters compared with existing approaches. Further research demonstrating clinical benefit is needed and ongoing. Further, the feasibility of long-term or continuous use of EIT for mechanically ventilated patients needs to be assessed, and head-to-head comparisons with existing methods of mechanical ventilation titration in humans need to be conducted. Although still in its adolescence, EIT may prove to be a useful bedside tool in the ICU in the near future.

\section{REFERENCES}

1. Adler A, Amato MB, Arnold JH, Bayford R, Bodenstein M, Bohm $\mathrm{SH}$, et al. Whither lung EIT: where are we, where do we want to go and what do we need to get there? Physiol Meas 2012;33(5):679694.

2. Techner E, Imhoff M, Leonhardt S. Electrical impedance tomography: the realization of regional ventilation monitoring. Draeger Marketing Materials 2011;129. http://www.draeger.com/sites/assets/ PublishingImages/Generic/UK/Booklets/rsp_eit_booklet_9066788_ en.pdf. Accessed June 16, 2016.

3. Slutsky AS, Ranieri VM. Ventilator-induced lung injury. N Engl J Med 2014;370(10):980.

4. Slutsky AS, Ranieri VM. Ventilator-induced lung injury. N Engl J Med 2013;369(22):2126-2136.

5. Frerichs I, Schmitz G, Pulletz S, Schädler D, Zick G, Scholz J, Weiler N. Reproducibility of regional lung ventilation distribution determined by electrical impedance tomography during mechanical ventilation. Physiol Meas 2007;28(7):S261-S267.

6. Wolf GK, Grychtol B, Frerichs I, van Genderingen HR, Zurakowski D, Thompson JE, Arnold JH. Regional lung volume changes in children with acute respiratory distress syndrome during a derecruitment maneuver. Crit Care Med 2007;35(8):1972-1978.

7. Hinz J, Hahn G, Neumann P, Sydow M, Mohrenweiser P, Hellige G, Burchardi H. End-expiratory lung impedance change enables bedside monitoring of end-expiratory lung volume change. Intensive Care Med 2003;29(1):37-43.

8. Frerichs I, Schiffmann H, Oehler R, Dudykevych T, Hahn G, Hinz J, Hellige G. Distribution of lung ventilation in spontaneously breath- ing neonates lying in different body positions. Intensive Care Med 2003;29(5):787-794.

9. Victorino JA, Borges JB, Okamoto VN, Matos GF, Tucci MR, Caramez MP, et al. Imbalances in regional lung ventilation: a validation study on electrical impedance tomography. Am J Respir Crit Care Med 2004;169(7):791-800.

10. Fagerberg A, Stenqvist O, Aneman A. Electrical impedance tomography applied to assess matching of pulmonary ventilation and perfusion in a porcine experimental model. Crit Care 2009;13(2):R34.

11. Reinius H, Borges JB, Fredén F, Jideus L, Camargo ED, Amato MB, et al. Real-time ventilation and perfusion distributions by electrical impedance tomography during one-lung ventilation with capnothorax. Acta Anaesthesiol Scand 2015;59(3):354-368.

12. Nguyen DT, Thiagalingam A, Bhaskaran A, Barry MA, Pouliopoulos J, Jin C, McEwan AL. Electrical impedance tomography for assessing ventilation/perfusion mismatch for pulmonary embolism detection without interruptions in respiration. Conf Proc IEEE Eng Med Biol Soc 2014;2014:6068-6071.

13. Costa EL, Chaves CN, Gomes S, Beraldo MA, Volpe MS, Tucci MR, et al. Real-time detection of pneumothorax using electrical impedance tomography. Crit Care Med 2008;36(4):1230-1238.

14. Schultz MJ. Electrical impedance tomography: a new toy for boys or the future for mechanically ventilated patients? Crit Care Med 2008; 36(4):1380-1381.

15. Kunst PW, Vazquez de Anda G, Böhm SH, Faes TJ, Lachmann B, Postmus PE, de Vries PM. Monitoring of recruitment and derecruitment by electrical impedance tomography in a model of acute lung injury. Crit Care Med 2000;28(12):3891-3895.

16. Hickling KG. The pressure-volume curve is greatly modified by recruitment. A mathematical model of ARDS lungs. Am J Respir Crit Care Med 1998;158(1):194-202.

17. Gattinoni L, D'Andrea L, Pelosi P, Vitale G, Pesenti A, Fumagalli R. Regional effects and mechanism of positive end-expiratory pressure in early adult respiratory distress syndrome. JAMA 1993;269(16): 2122-2127.

18. Keenan JC, Dries DJ. PEEP titration: new horizons. Respir Care 2013;58(9):1552-1554.

19. Villar J, Pérez-Méndez L, López J, Belda J, Blanco J, Saralegui I, et al. An early $\mathrm{PEEP} / \mathrm{F}_{\mathrm{IO}_{2}}$ trial identifies different degrees of lung injury in patients with acute respiratory distress syndrome. Am J Respir Crit Care Med 2007;176(8):795-804.

20. Kallet RH, Branson RD. Respiratory controversies in the critical care setting. Do the NIH ARDS Clinical Trials Network PEEP/F $\mathrm{F}_{\mathrm{IO}_{2}}$ tables provide the best evidence-based guide to balancing PEEP and $\mathrm{F}_{\mathrm{IO}_{2}}$ settings in adults? Respir Care 52(4):461-475, 2007; discussion 475467.

21. Pulletz S, Adler A, Kott M, Elke G, Gawelczyk B, Schadler D, et al. Regional lung opening and closing pressures in patients with acute lung injury. J Crit Care 2012;27(3):323.e311-e328.

22. Wolf GK, Gómez-Laberge C, Kheir JN, Zurakowski D, Walsh BK, Adler A, Arnold JH. Reversal of dependent lung collapse predicts response to lung recruitment in children with early acute lung injury. Pediatr Crit Care Med 2012;13(5):509-515.

23. Costa EL, Lima RG, Amato MB. Electrical impedance tomography. Curr Opin Crit Care 2009;15(1):18-24.

24. Drakulovic MB, Torres A, Bauer TT, Nicolas JM, Nogué S, Ferrer M. Supine body position as a risk factor for nosocomial pneumonia in mechanically ventilated patients: a randomised trial. Lancet 1999; 354(9193):1851-1858.

25. Rose L, Baldwin I, Crawford T, Parke R. Semirecumbent positioning in ventilator-dependent patients: a multicenter, observational study. Am J Crit Care 2010;19(6):e100-e108.

26. Spooner AJ, Corley A, Sharpe NA, Barnett AG, Caruana LR, Hammond NE, Fraser JF. Head-of-bed elevation improves end-expiratory 


\section{Electrical Impedance Tomography During Mechanical Ventilation}

lung volumes in mechanically ventilated subjects: a prospective observational study. Respir Care 2014;59(10):1583-1589.

27. Bein T, Ploner F, Ritzka M, Pfeifer M, Schlitt HJ, Graf BM. No change in the regional distribution of tidal volume during lateral posture in mechanically ventilated patients assessed by electrical impedance tomography. Clin Physiol Funct Imaging 2010;30(4):234240.

28. Mauri T, Bellani G, Confalonieri A, Tagliabue P, Turella M, Coppadoro A, et al. Topographic distribution of tidal ventilation in acute respiratory distress syndrome: effects of positive end-expiratory pressure and pressure support. Crit Care Med 2013;41(7):1664-1673.

29. Blankman P, Hasan D, van Mourik MS, Gommers D. Ventilation distribution measured with EIT at varying levels of pressure support and neurally adjusted ventilatory assist in patients with ALI. Intensive Care Med 2013;39(6):1057-1062.

30. Yoshida T, Torsani V, Gomes S, De Santis RR, Beraldo MA, Costa EL, et al. Spontaneous effort causes occult pendelluft during mechanical ventilation. Am J Respir Crit Care Med 2013;188(12):14201427.

31. Yoshida T, Uchiyama A, Matsuura N, Mashimo T, Fujino Y. The comparison of spontaneous breathing and muscle paralysis in two different severities of experimental lung injury. Crit Care Med 2013; 41(2):536-545.

32. Papazian L, Forel JM, Gacouin A, Penot-Ragon C, Perrin G, Loundou A, et al. Neuromuscular blockers in early acute respiratory distress syndrome. N Engl J Med 2010;363(12):1107-1116.

33. Bhatia R, Schmölzer GM, Davis PG, Tingay DG. Electrical impedance tomography can rapidly detect small pneumothoraces in surfactant-depleted piglets. Intensive Care Med 2012;38(2):308-315.

34. Miedema M, Frerichs I, de Jongh FH, van Veenendaal MB, van Kaam AH. Pneumothorax in a preterm infant monitored by electrical impedance tomography: a case report. Neonatology 2011;99(1):1013.

35. Costa EL, Borges JB, Melo A, Suarez-Sipmann F, Toufen C Jr, Bohm SH, Amato MB. Bedside estimation of recruitable alveolar collapse and hyperdistension by electrical impedance tomography. Intensive Care Med 2009;35(6):1132-1137.

36. Dargaville PA, Rimensberger PC, Frerichs I. Regional tidal ventilation and compliance during a stepwise vital capacity manoeuvre. Intensive Care Med 2010;36(11):1953-1961.

37. Frerichs I, Achtzehn U, Pechmann A, Pulletz S, Schmidt EW, Quintel $\mathrm{M}$, Weiler N. High-frequency oscillatory ventilation in patients with acute exacerbation of chronic obstructive pulmonary disease. $\mathbf{J}$ Crit Care 2012;27(2):172-181.

38. Gómez-Laberge C, Arnold JH, Wolf GK. A unified approach for EIT imaging of regional overdistension and atelectasis in acute lung injury. IEEE Trans Med Imaging 2012;31(3):834-842.

39. Pulletz S, Kott M, Elke G, Schädler D, Vogt B, Weiler N, Frerichs I. Dynamics of regional lung aeration determined by electrical impedance tomography in patients with acute respiratory distress syndrome. Multidiscip Respir Med 2012;7(1):44.

40. Zhao Z, Steinmann D, Frerichs I, Guttmann J, Möller K. PEEP titration guided by ventilation homogeneity: a feasibility study using electrical impedance tomography. Crit Care 2010;14(1):R8.

41. Zhao Z, Steinmann D, Müller-Zivkovic D, Martin J, Frerichs I, Guttmann J, Möller K. A lung area estimation method for analysis of ventilation inhomogeneity based on electrical impedance tomography. J Xray Sci Technol 2010;18(2):171-182.

42. Wolf GK, Gómez-Laberge C, Rettig JS, Vargas SO, Smallwood CD, Prabhu SP, et al. Mechanical ventilation guided by electrical impedance tomography in experimental acute lung injury. Crit Care Med 2013;41(5):1296-1304

43. Grasso S, Stripoli T, De Michele M, Bruno F, Moschetta M, Angelelli $\mathrm{G}$, et al. ARDSnet ventilatory protocol and alveolar hyperinflation: role of positive end-expiratory pressure. Am J Respir Crit Care Med 2007;176(8):761-767.

44. Loring SH, Weiss JW. Plateau pressures in the ARDSnet protocol: cause of injury or indication of disease? Am J Respir Crit Care Med 176(1):99-100, 2007; author reply 100-101.

45. de Durante G, del Turco M, Rustichini L, Cosimini P, Giunta F, Hudson LD, et al. ARDSNet lower tidal volume ventilatory strategy may generate intrinsic positive end-expiratory pressure in patients with acute respiratory distress syndrome. Am J Respir Crit Care Med 2002;165(9):1271-1274

46. Slutsky AS, Ranieri VM. Mechanical ventilation: lessons from the ARDSNet trial. Respir Res 2000;1(2):73-77.

47. Snashall PD, Keyes SJ, Morgan BM, McAnulty RJ, Mitchell-Heggs PF, McIvor JM, Howlett KA. The radiographic detection of acute pulmonary oedema. A comparison of radiographic appearances, densitometry and lung water in dogs. Br J Radiol 1981;54(640):277-288.

48. Trepte CJ, Phillips CR, Solà J, Adler A, Haas SA, Rapin M, et al. Electrical impedance tomography (EIT) for quantification of pulmonary edema in acute lung injury. Crit Care 2016;20(1):18. 